\title{
Early impairment of large artery structure and function in Type I diabetes mellitus
}

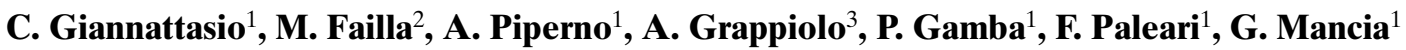 \\ ${ }^{1}$ Department of Internal Medicine, S. Gerardo Hospital, Monza, Italy \\ ${ }^{2}$ IRCSS Italian Auxologic Centre, Milan, Italy \\ ${ }^{3}$ IRCSS Centre of Clinical Physiology and Hypertension, Maggiore Hospital, Milan, Italy
}

\section{Abstract}

Aims/hypothesis. Diabetes mellitus is associated with an increased incidence of atherosclerosis. How early functional and structural alterations of large arteries that may preceed atherosclerosis occur in the course of this disease has, however, never been conclusively documented.

Methods. We evaluated arterial wall distensibility in the radial artery, common carotid artery and abdominal aorta in 133 patients (aged $35.4 \pm 0.9$ years, means \pm SEM) with Type I (insulin-dependent) diabetes mellitus and no macrovascular complications. Arterial distensibility was derived from continuous measurements of arterial diameter through echotracking techniques and use of either the Langewouters (radial artery) or the Reneman (carotid artery and aorta) formula. The same echotracking techniques enabled us to obtain radial artery and carotid artery wall thickness. Data were compared with those from 70 age-matched normotensive control subjects.

Results. In diabetic patients arterial distensibility was consistently less $(p<0.01)$ than in control subjects, the reduction averaging $26 \%, 14 \%$ and $25 \%$ for the radial artery, carotid artery and aorta, respectively.
This was accompanied by an increase $(p<0.01)$ in both radial and carotid artery wall thickness. The changes were more pronounced in patients with microalbuminuria, retinopathy or neuropathy or both. They were evident also in those without microvascular complications. This was the case also when subjects in whom diabetes was associated with hypertension $(n=30)$ were excluded from data analysis. Carotid and aortic wall abnormalities showed a relation with the duration of disease and blood pressure whereas radial artery abnormalities showed a relation with glycated haemoglobin.

Conclusion/interpretation. Type I diabetes is characterised by diffuse arterial wall stiffening and thickening which progress with the severity of the disease but can clearly be seen also in the absence of any diabetic-related complication. This suggests that in diabetes stiffening and thickening are an early marker of vascular damage. [Diabetologia (1999) 42: 987994]

Keywords Type I diabetes mellitus, arterial wall distensibility, arterial wall structure, atherosclerosis, microalbuminuria, diabetic complications, hypertension.
Diabetes mellitus is associated with an increased risk of atherosclerosis [1]. Atherosclerosis is responsible for the increased rate of coronary heart disease, cere-

Received: 11 December 1998 and in revised form: 3 March 1999

Corresponding author: Professor G. Mancia, Clinica Medica, Università di Milano, Ospedale S.Gerardo dei Tintori, V. Donizetti 106, Monza (MI), Italy brovascular disease and peripheral artery disease typical of diabetes mellitus [2-13]. It is not, however, well established how early alterations in large artery function, that may preceed the appearance and favour the progression of atherosclerotic lesions, i.e. a reduced arterial distensibility, occur in diabetic patients [14-16]. This is because most studies on arterial distensibility have been done in Type II (non-insulindependent) diabetes mellitus, where it is difficult to separate the arterial stiffening due to abnormalities 
of glucose metabolism from that due to age and associated diseases [17-23]. Furthermore, the few studies that have measured arterial distensibility in Type I (insulin-dependent) diabetes mellitus (i.e. a condition in which the age of the disease can be more easily identified and concomitant confounding factors excluded) have been done not only on a small number of patients but have reported both a reduction and an increase in arterial distensibility thereby reaching opposite conclusions [24-28].

Our study was carried out to systematically address this issue in a large number of patients with Type I diabetes. To obtain data that could be representative of the whole arterial tree, distensibility was measured in muscular or elastic vessels of different sizes. Measurements included arterial wall thickness to determine whether changes in large artery function are accompanied by structural changes.

\section{Methods}

Subjects. We investigated 203 subjects. We selected 133 subjects ( 74 men; aged $35.4 \pm 0.9$ years) on a consecutive basis if they had: 1) Type I diabetes, were receiving insulin treatment and had good metabolic control, 2) no evidence of clinical or subclinical atherosclerotic disease in their history at physical or laboratory examination such as a chest radiograph, standard and exercise ECG, an echocardiogram and an echocolor-Doppler of carotid arteries, femoral arteries and abdominal aorta, 3) no major disease except diabetes and no long-term treatment with other drugs. The remaining 70 subjects (48 men; 22 women; aged $33.8 \pm 1.1$ years) were age-matched normotensive healthy people who were used as controls. All subjects agreed to participate in the study after being informed of its nature and purpose. The protocol of the study was approved by the ethics committees of the institutions involved.

Radial artery distensibility and wall thickness. Radial artery diameter and wall thickness were measured by an A-mode ultrasonic device (NIUS 02, Omega, Bienne, Switzerland and Capital Medical Services Paris, France) [29]. Briefly, a highly focalised transducer operating at a frequency of $10 \mathrm{MHz}$ was stereotaxically positioned over the radial artery $2-4 \mathrm{~cm}$ above the wrist, direct contact with the skin being prevented by use of a gel as a medium. With the subject supine and the arm immobile at heart level, the transducer was oriented perpendicularly to the longitudinal axis of the vessel based on the acoustic Doppler signal. After switching to A-mode the echo beams corresponding to the posterior lumen-intima and media-adventitia interfaces were made visible on a computer screen $[29,30]$ and the wall thickness of the artery was measured [30] as the distance between the inner and outer wall echoes. The backscattered echoes from the inner posterior and anterior walls of the artery were also visualised on the computer screen and electronically digitised (via an analogic/digital fast transducer) to allow internal diameter variations to be derived at a $50 \mathrm{~Hz}$ with a spatial resolution of $0.0025 \mathrm{~mm}[29,30]$.

The device also made use of a photoplethysmographic system (Finapres 2003, Ohmeda, Englewood, Colo., USA) which allowed blood pressure to be recorded at $50 \mathrm{~Hz}$ from a finger ipsilateral to the radial artery examined, with an accuracy sim- ilar to intra-arterial blood pressure recording [31] and a resolution of $2 \mathrm{mmHg}$ [31]. The concomitant acquisition of continuous arterial diameter and blood pressure signals allowed us to calculate the diameter across the diasto-systolic pressure range. The diameter pressure curve was analysed according to its fitting with the arctangent model of Langewouters which is based on the formula:

$S=\alpha\left[\frac{\pi}{2}+\tan ^{-1}\left(\frac{P-\beta}{\gamma}\right)\right]$

where $S$ is the cross-sectional area, $P$ is blood pressure and $\alpha, \beta$ and $\gamma$ are three optimal variables describing the spatial position of the diameter/pressure curve [32].

Cross-sectional compliance $(C=\Delta \mathrm{S} / \Delta \mathrm{P})[33]$ was calculated as:

$C=\frac{\alpha}{\gamma} \frac{1}{1+\left(\frac{P-\beta}{\gamma}\right)^{2}}$

The same formula was used to calculate cross-sectional distensibility (cross-sectional compliance divided by vessel area)/ pressure curve.

Carotid artery distensibility and wall thickness. With the subject supine and the neck in partial extension, the diameter and wall motion of the right common carotid artery were measured $2 \mathrm{~cm}$ below the carotid bifurcation by a B-M mode echo-tracking device based on Doppler shift (Wall Track System, PIE Medical, Maastricht, The Netherlands) and on a transducer operating at a frequency of $7.5 \mathrm{MHz}[34,35]$. The transducer was manually oriented perpendicularly to the longitudinal axis of the vessel under B-mode guidance. After switching to A-mode the backscattered echoes from the anterior and posterior carotid artery walls were made visible on a screen and the corresponding radiofrequency signal was tracked by electronic tracers to allow the digitalised signal of the internal diameter variations to be derived at $50 \mathrm{~Hz}$. The spatial resolution was $300 \mu \mathrm{m}$ [36]. Blood pressure was measured from the brachial artery at the same time as the ultrasound evaluation by a semiautomatic device (Dinamap 1846 SX/SXP, Critikon, Chatenay Malabry Cedex, France) and carotid artery distensibility was derived according to the following formula [34]:

Dist $=(2 \Delta \mathrm{D} / \mathrm{Dd}) / \Delta \mathrm{P}$

where Dist was distensibility, Dd the diastolic diameter of the vessel, $\Delta \mathrm{D}$ the systo-diastolic diameter change and $\Delta \mathrm{P}$ the corresponding pulse pressure.

Carotid artery intima-media wall thickness was measured at a posterior wall site located $3 \mathrm{~cm}$ below bifurcation through an ultrasonographic device (Hewlett Packard Sonos 5500, Palo Alto, Calif., USA). Measurements were obtained by first scanning the artery in B-Mode, then freezing the digitised image in M-mode and finally tracking the inner ipoechogenic and the middle anechogenic layers [37].

Aortic distensibility. With the subject supine the diameter and wall motion of the subdiaphragmatic portion of the aorta were measured in 189 subjects (127 Type I diabetic patients and 62 control subjects), $2-3 \mathrm{~cm}$ above the origin of the celiac plexus, by the same B-M mode echo-tracking device used for the carotid artery $[34,35]$. The transducer (which operated at a frequency of $3.5 \mathrm{MHz}$ ) was manually oriented perpendicularly to the longitudinal axis of the vessel under B-mode guidance, and brachial artery blood pressure was measured at the 
Table 1. Demographic, haemodynamic and metabolic findings in control subjects and Type I diabetic patients without and with microvascular complications

\begin{tabular}{lccr}
\hline & $\begin{array}{l}\text { Control subjects } \\
(n=70)\end{array}$ & $\begin{array}{l}\text { Type I diabetic } \\
\text { without complications } \\
(n=54)\end{array}$ & $\begin{array}{l}\text { Type I diabetic } \\
\text { with complications } \\
(n=79)\end{array}$ \\
\hline Body surface area $\left(\mathrm{m}^{2}\right)$ & $1.8 \pm 0.02$ & $1.7 \pm 0.02^{\mathrm{a}}$ & $1.7 \pm 0.02^{\mathrm{a}}$ \\
Systolic BP (mm Hg) & $127.5 \pm 1.6$ & $128.2 \pm 1.5$ & $145.4 \pm 2.4^{\mathrm{b}, \mathrm{d}}$ \\
Diastolic BP (mm Hg) & $76.8 \pm 1.0$ & $75.7 \pm 0.9$ & $82.0 \pm 1.0^{\mathrm{a}, \mathrm{c}}$ \\
Heart rate (beats/min) & $71.0 \pm 1.4$ & $75.9 \pm 1.4^{\mathrm{a}}$ & $75.8 \pm 1.3^{\mathrm{a}}$ \\
Serum glucose (mmol/l) & $/$ & $8.9 \pm 0.4$ & $9.7 \pm 0.5$ \\
Duration of diabetes (years) & $/$ & $12.0 \pm 1.1$ & $16.7 \pm 0.9^{\mathrm{d}}$ \\
HbA $_{1 \mathrm{c}}$ (\%) & $/$ & $4.9 \pm 0.2$ & $8.5 \pm 0.2^{\mathrm{c}}$ \\
Insulin (U/day) & $43.2 \pm 2.2$ & $42.6 \pm 1.4$ \\
\hline
\end{tabular}

Data are shown as means $\pm \mathrm{SEM} ;{ }^{\mathrm{a}} p<0.05$ and ${ }^{\mathrm{b}} p<0.01$ vs control; ${ }^{\mathrm{c}} p<0.05,{ }^{\mathrm{d}} p<0.01$ vs diabetic without complications

same time as the ultrasound evaluation by the same semiautomatic device used for the carotid artery. For the carotid artery, aortic distensibility was derived from the Reneman formula. For technical reasons no measurement of aortic wall thickness could be made systematically.

All radial, carotid and aortic measurements were made by a single operator. The within-operator variability of radial artery, carotid artery and aortic diameter measurements at diastole (i.e. the coefficient of variation of the mean values of two measurements done at two different times) was on average $2.5 \%, 3.5 \%$ and $4.5 \%$, respectively. The within operator variability for radial artery and for carotid artery wall thickness were $3.0 \%$ and $4.0 \%$, respectively.

Additional evaluations. Blood pressure was measured not only by the finger pressure or the Dinamap device (see above) but also by a mercury sphygmomanometer, taking the first and fifth Korotkoff sounds to identify systolic and diastolic values, respectively. Heart rate was obtained continuously from the finger pressure signal as the reciprocal of the pulse interval between consecutive beats. In diabetic patients serum glucose and glycated haemoglobin values were derived from data collected within the preceeding 3 months. Based on three overnight microalbuminuria [38] measurements, an optic fundus and an electromyographic examination available in the preceeding 9 months [39] 54 patients (aged $32.2 \pm 1.3$ years) were found to be devoid of microvascular complication, 29 to have incipient nephropathy, 44 retinopathy and 24 neuropathy. The number of patients with at least one microvascular complication was 79 (aged $37.6 \pm 1.2$ years). Hypertension (sphygmomanometric blood pressure above $140 \mathrm{mmHg}$ systolic or $90 \mathrm{mmHg}$ diastolic) was found in 30 patients all belonging to the group with microvascular complications.

Protocol and data analysis. Each patient was asked to come to the outpatient clinic of the San Gerardo Hospital in the afternoon after a 24-h abstinence from alcohol, caffeine consumption and cigarette smoking. The protocol of the study was as follows: 1) blood pressure was measured three times by a mercury sphygmomanometer with the patient in the sitting position, 2) the subject was placed in the supine position and fitted with finger blood pressure and radial artery echo-tracking devices, 3) after a 10-min interval finger blood pressure, heart rate and radial artery diameter were continuously measured for $15 \mathrm{~min}, 4)$ the radial artery echo-tracking device was disconnected, the semiautomatic blood pressure measuring device was placed on the brachial artery and the probe for carotid artery evaluation was positioned on the neck. 5) five 6-s acquisitions of carotid diameter throughout the cardiac cycle were obtained during a 10-min period together with semiautomatic blood pressure measurements, 6) the probe for evaluation of the abdominal aorta was positioned over the abdomen, 7) five 6-s acquisitions of the abdominal aortic diameter throughout the cardiac cycle were obtained during a 10-min period together with semiautomatic blood pressure measurements as for the carotid artery.

The three sphygmomanometric blood pressure values were averaged. Radial artery distensibility/pressure curves were obtained by averaging data from five periods of $30 \mathrm{~s}$ each, taken at intervals of $2 \mathrm{~min}$. The area under the radial artery distensibility/pressure curve was corrected for pulse pressure and used to obtain a single comprehensive distensibility value which was called the radial artery distensibility index. Radial artery diameter at diastole and the individual distensibility index were obtained by averaging values corresponding to diastolic blood pressure over the same five periods. Carotid artery diastolic diameter and distensibility were obtained by averaging data from the five 6 -s acquisition periods. The same procedure was adopted for aortic diastolic diameter and distensibility. Radial artery and carotid artery wall thickness were measured on the screen image of the vessels over a 30-s period, based on the ultrasound image.

Results from individual subjects were averaged and shown as means \pm SEM. The statistical significance of the differences in mean values was assessed by two-way analysis of variance. The two-tailed $t$ test for unpaired observations was used to locate differences between control subjects, diabetic subjects without and with microvascular complications (with and without hypertension) using the Bonferroni correction for multiple comparisons. Data were also analysed by univariate regression taking arterial distensibility or wall thickness as the dependent variables and patient's age, duration of disease, clinic systolic blood pressure and metabolic data as the independent ones. Significant correlations were then also tested by multivariate analysis. A $p$ value less than 0.05 was taken as statistically significant.

\section{Results}

Body surface area was slightly less in diabetic patients than in controls (Table 1). Sphygmomanometric systolic and diastolic blood pressure were similar in controls and diabetic patients without microvascular complications but slightly greater in diabetic patients with microvascular complications. Heart rate was slightly, although significantly $(p<0.05)$ greater in diabetic patients with or without microvascular compli- 

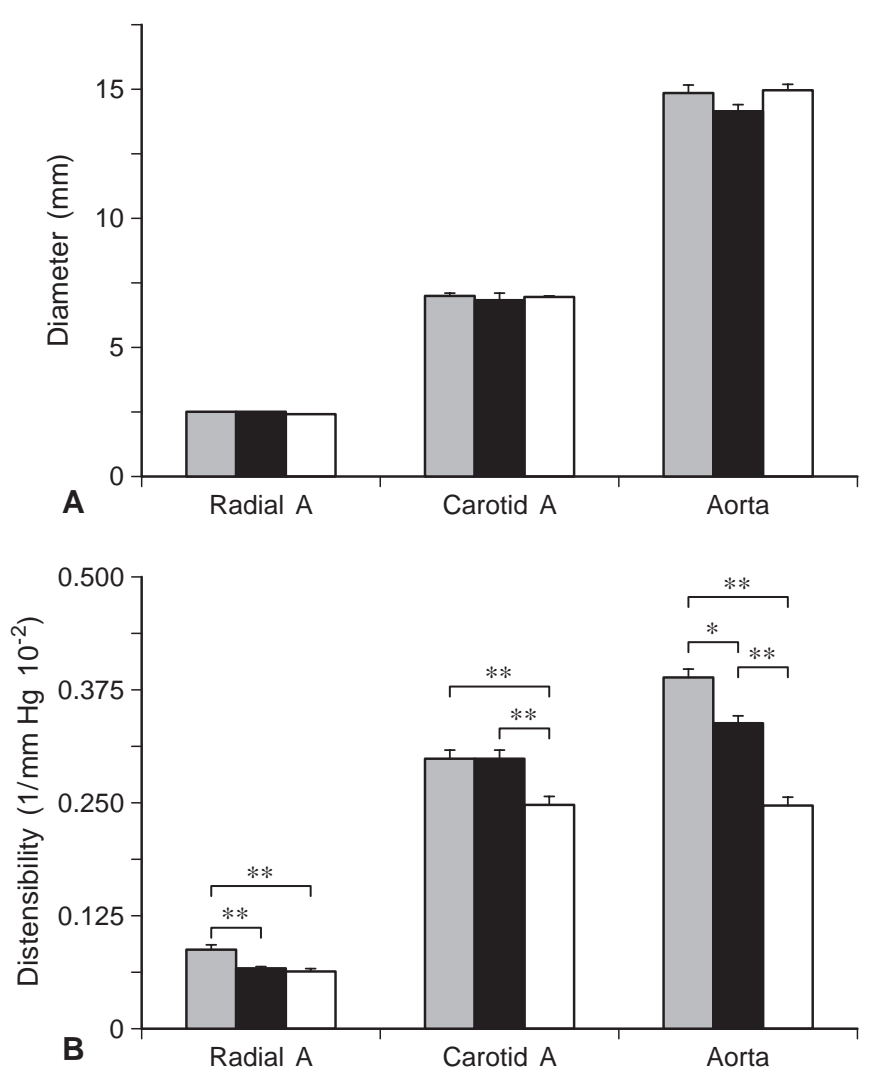

Fig.1. A Arterial diastolic diameters in control subjects (grey histograms, $n=70$ for radial and for carotid, $n=62$ for aorta) and subjects with Type I diabetes without (black histograms, $n=54$ for radial and for carotid, and $n=53$ for aorta) and with microvascular complications (white histograms, $n=79$ for radial and for carotid and 74 for aorta). B Arterial distensibility in the same three groups of subjects. Data are shown as means \pm SEM. Figures at top of each histogram refer to number of subjects in each group or subgroup. A: artery, ${ }^{*} p<0.05$; $* * p<0.01$

cations compared with control subjects. In diabetic patients with microvascular complications, duration of diabetes, serum glucose and glycated haemoglobin were greater than in diabetic patients without microvascular complications, whereas the daily dose of insulin was similar in the two groups.

In control subjects, diabetic patients without microvascular complications and diabetic patients with microvascular complications, arterial diameter and distensibility were progressively greater from the radial artery to the carotid artery and the aorta (Fig.1). Arterial diameters were consistently similar in the three groups. Compared with control subjects, however, carotid artery distensibility was unchanged in diabetic patients without but reduced in diabetic patients with microvascular complications. Radial artery and aortic distensibility were reduced in diabetic patients without microvascular complications, those with microvascular complications showing a further reduction. The progressive reduction in radial artery distensibility from control subjects to diabetic pa-

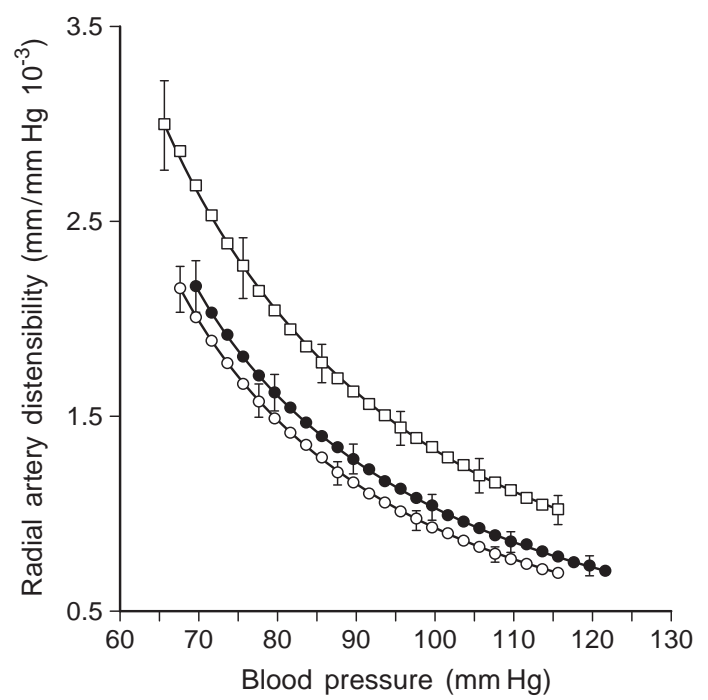

Fig. 2. Radial artery distensibility/ blood pressure curves in control subjects and diabetic patients with no microvascular complications and diabetic subjects with microvascular complications. $\square$ Control subjects $(n=70) \bigcirc$ no complications $(n=59) \bigcirc$ with complications $(n=74)$

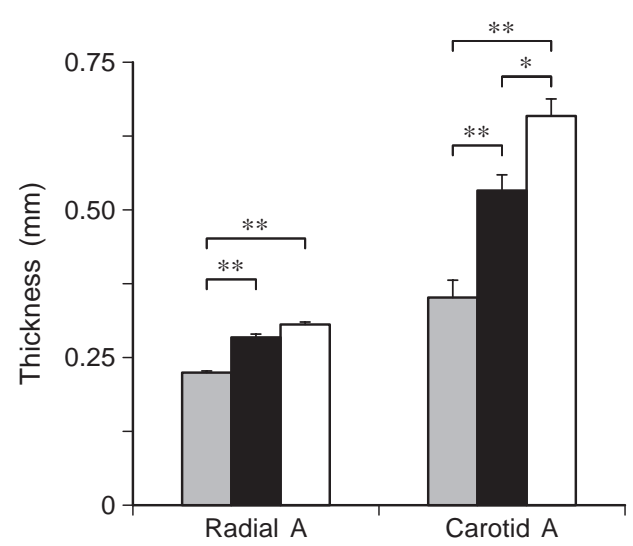

Fig.3. Arterial wall thickness in control subjects (grey histograms, $n=70$ for radial and for carotid) and diabetic patients with no microvascular complications (black histograms, $n=54$ for radial and for carotid) and diabetic patients with microvascular complications (white histograms $n=79$ for radial and for carotid). A: artery, ${ }^{*} p<0.05 ; * * p<0.01$

tients without and with microvascular complications was visible throughout the diasto-systolic blood pressure range (Fig. 2). When all subjects were pooled, a relation was found between carotid artery and aortic distensibility $(r=0.57 ; p<0.05)$ but not between carotid artery or aortic distensibility and radial artery distensibility ( $r=0.16$ and 0.09 , respectively, NS).

For both the radial and the carotid artery, wall thickness was greater in diabetic patients without microvascular complications than in control subjects, diabetic patients with microvascular complications showing a trend (significant for the carotid artery) towards a greater change (Fig. 3). In this group the arte- 
Table 2. Blood pressure and large artery wall findings in control subjects and Type I normotensive patients (pts)

\begin{tabular}{lccc}
\hline Variable & $\begin{array}{l}\text { Control subjects } \\
(n=10)\end{array}$ & $\begin{array}{l}\text { Normotensive } \\
\text { Type I diabetic } \\
\text { with complications } \\
(n=49)\end{array}$ & $\begin{array}{l}\text { Hypertensive } \\
\text { Type I diabetic } \\
\text { with complications } \\
(n=30)\end{array}$ \\
\hline Systolic blood pressure $(\mathrm{mm} \mathrm{Hg})$ & $127.5 \pm 1.6$ & $131.7 \pm 1.8$ & $78.0 \pm 1.0$ \\
Diastolic blood pressure $(\mathrm{mm} \mathrm{Hg})$ & $76.8 \pm 1.0$ & $0.30 \pm 0.01^{\mathrm{b}}$ & $164.5 \pm 3.0^{\mathrm{b}, \mathrm{c}}$ \\
Radial artery wall thickness $(\mathrm{mm})$ & $0.22 \pm 0.01$ & $0.59 \pm 0.02^{\mathrm{b}}$ & $87.5 \pm 1.5^{\mathrm{a}}$ \\
Carotid artery wall thickness $(\mathrm{mm})$ & $0.35 \pm 0.03$ & $0.67 \pm 0.04^{\mathrm{b}}$ & $0.30 \pm 0.01^{\mathrm{b}}$ \\
Radial artery distensibility $\left(1 / \mathrm{mm} \mathrm{Hg} 10^{-3}\right)$ & $0.88 \pm 0.05$ & $0.28 \pm 0.01^{\mathrm{b}}$ & $0.71 \pm 0.06^{\mathrm{b}}$ \\
Carotid artery distensibility $\left(\mathrm{mm} / \mathrm{mm} \mathrm{Hg} 10^{-2}\right)$ & $0.30 \pm 0.01$ & $0.29 \pm 0.01^{\mathrm{b}}$ & $0.20 \pm 0.05^{\mathrm{b}}$ \\
Aortic distensibility $\left(\mathrm{mm} / \mathrm{mm} \mathrm{Hg} 10^{-2}\right)$ & $0.38 \pm 0.01$ & $0.21 \pm 0.01^{\mathrm{b}}$ \\
\hline
\end{tabular}

Data are shown as means \pm SEM; ${ }^{a} p<0.05$ and ${ }^{b} p<0.01$ vs control subjects ${ }^{c} p<0.05$ vs diabetic normotensive without complications

Table 3. Univariate regressions between arterial wall structure or distensibility (dependent variables) and the independent variables indicated in vertical column

\begin{tabular}{|c|c|c|c|c|c|}
\hline & RA distensibility & CA distensibility & Aortic distensibility & RA wall thickness & CA wall thickness \\
\hline \multicolumn{6}{|l|}{ Age } \\
\hline$r$ & 0.10 & -0.59 & -0.59 & 0.15 & 0.52 \\
\hline$p<$ & NS & 0.0001 & 0.0001 & NS & 0.0001 \\
\hline$r$ & 0.04 & -0.49 & -0.39 & 0.04 & 0.37 \\
\hline$p<$ & NS & 0.0001 & 0.0001 & NS & 0.0001 \\
\hline \multicolumn{6}{|c|}{ Systolic blood pressure } \\
\hline$p<$ & NS & 0.0001 & 0.0001 & NS & 0.0001 \\
\hline \multicolumn{6}{|c|}{$\mathrm{HbA}_{1 \mathrm{c}} \%$} \\
\hline$r$ & -0.14 & 0.04 & 0.04 & 0.26 & 0.07 \\
\hline$p<$ & NS & NS & NS & 0.05 & NS \\
\hline \multicolumn{6}{|c|}{ Glycaemia } \\
\hline$r$ & -0.149 & 0.025 & 0.12 & 0.143 & 0.126 \\
\hline
\end{tabular}

RA: radial artery; CA: carotid artery

rial distensibility was slightly less and wall thickness slightly greater in subjects with hypertension than in subjects with normal blood pressure (Table 2). Radial and carotid artery wall thicknesses showed a weak correlation $(r=0.30, p<0.05)$ and each of these values also showed a weak correlation with the corresponding vessel distensibility (radial, $r=0.22$; carotid, $r=0.35, p<0.05$ for both).

In diabetic patients the carotid artery and aortic distensibility were inversely related to patient's age, duration of diabetes and blood pressure, all of which were directly related to carotid wall thickness (Table 3). In contrast, none of these variables had a relation with radial artery distensibility and wall thickness which were, however, related $(p<0.05)$, to glycated haemoglobin. No statistically significant relation was seen between arterial distensibility and wall thickness values and other measures of metabolic disturbances such as serum glucose and the daily dose of insulin. The variables that were related to carotid ar- tery distensibility, aortic distensibility and carotid artery wall thickness (age, duration of diabetes and systolic blood pressure) maintained a statistically significant relation in a multivariable analysis including all related variables (Table 4).

\section{Discussion}

In our Type I diabetic patients with an average age of about 35 years and no macrovascular complications, radial artery, carotid artery and aortic distensibility were all reduced compared with age-matched control subjects but carotid and radial artery wall thickness were increased. Furthermore, with the exception of carotid artery distensibility, all changes were visible in diabetic patients with no clinical evidence of microvascular complications (and thus with no diabetic complications of any kind), although their magnitude increased with the duration of disease and was defi- 
Table 4. Multivariate regression between arterial wall structure and distensibility and the independent variables indicated in vertical column

\begin{tabular}{llll}
\hline & CA distensibility & Aortic distensibility & CA wall thickness \\
\hline Age & beta: $-0.004, p<0.01$ & beta: $-0.006, p<0.01$ & beta: $0.01, p<0.01$ \\
Duration of diabetes & beta: $-0.002, p<0.04$ & beta: $-0.003, p<0.05$ & beta: $0.02, p<0.05$ \\
Systolic blood pressure & beta: $-0.001, p<0.01$ & beta: $-0.003, p<0.01$ & beta: 0.02, NS \\
\hline
\end{tabular}

CA: carotid artery

nitely more pronounced in those with microvascular complications. Finally, the alterations in arterial distensibility and wall thickness were not limited to diabetic hypertensive patients but were observed in diabetic normotensive patients as well. It is thus possible to conclude that Type I diabetes mellitus is characterised by diffuse alterations in arterial function and structure. These alterations progress with the increasing severity of the disease. In most arteries, they can, however, be seen clearly without any evidence of diabetic-related complications and blood pressure abnormalities, which unequivocally shows that in diabetes generalised vascular abnormalities are a very early subclinical phenomenon.

Our results do not clarify the mechanisms responsible for the reduction of arterial distensibility that characterises Type I diabetes mellitus. Because this reduction occurred, however, together with and in relation to an increase in arterial wall thickness, an alteration in arterial wall structure is presumably involved. This alteration could originate from the reduced nitric oxide secretion that can be seen in diabetes [39], because firstly nitric oxide has been shown to oppose proliferation of vessel wall tissue in vitro [40] and secondly endothelial function has been found to be inversely related to carotid artery wall thickness both in studies of animals and humans in vivo [41, 42]. It could, however, also be due to the insulin given exogeneously because insulin is known to be a growing factor for vascular wall tissues [43]. Finally it could be due to the intravascular pressure load because mechanical stretching of a vessel wall also stimulates its tissue components to undergo cell replication and growth [44]. Indeed, all these factors are likely to be involved because markers of altered glucose metabolism (which negatively modulates nitric oxide secretion) [45], daily insulin doses and blood pressure all showed some correlation with arterial distensibility and wall thickness. Although carotid artery and aortic distensibility and wall thickness correlated with blood pressure but not with glycated haemoglobin, this was, however, reversed for radial artery distensibility. This suggests that there could be a heterogeneity in the factors that cause vascular functional and structural changes in diabetes, i. e. that mechanical influences may have a greater role in larger arteries whereas medium size arteries may be more protected from blood pressure-related trauma but subjected to factors deranging glucose metabolism and increasing vascular tissue growth [43-46]. This with the caveat that firstly correlation data may not represent a cause-effect relation and secondly several other factors potentially involved in alterations of vascular wall structure were not measured in our study.

Other aspects of our study are firstly that our assessment of radial artery distensibility was accurate because blood pressure was measured beat-to-beat at a site near the one where arterial diameter changes were tracked, providing distensibility-pressure curves that are superimposable to those obtained by intraarterial monitoring from the radial artery itself [47]. On the other hand, assessment of aortic and carotid artery distensibility was less precise because blood pressure could only be taken from the brachial artery, i.e. at a peripheral site where pulse pressure is slightly amplified and thus the size of the stimulus distending the vessel wall overestimated. This approach is, however, currently used in arterial distensibility studies. Furthermore, in our study any possible error of overestimation was presumably similar in control subjects and normotensive diabetic patients, with no substantial influence on their difference.

Secondly, the relation between arterial distensibility and wall thickness, although statistically significant, was not close, suggesting that in diabetes alterations of arterial distensibility can also occur through a functional adverse effect on arterial mechanical properties. Endothelial dysfunction occurring in diabetes could also be involved because a reduced nitric oxide secretion may lead to an increased state of contraction of smooth muscle in the vessel wall [48], increasing the vessel elastic modulus compared with muscle in the relaxed state.

Thirdly, alterations in carotid artery distensibility were significantly $(p<0.05)$ but not closely related to alterations in aortic distensibility and in both instances little or no relation with radial artery distensibility was observed. This confirms previous findings in other diseases that alterations in large artery mechanical properties can be heterogeneous throughout the arterial tree $[49,50]$. Namely, that large elastic arteries can behave differently from middle-sized muscular vessels and that therefore proper characterisation of arterial mechanical characteristics need to be done at multiple sites.

Fourthly, our results suggest that attention should be given to vascular mechanical and anatomical prop- 
erties in diabetic patients to uncover abnormalities with an atherogenic relevance when no clinical complications of diabetes are apparent. It should be emphasised, however, that this implies the availability of complex and expensive technology which may be beyond the means of most hospitals.

Finally, it is important to emphasise that both a reduced arterial distensibility and an increased arterial wall thickness have a clear-cut pathophysiological relevance. This is because an increased arterial wall thickness may lead the less vascularised wall layer, i.e. the intima, to receive less oxygen and other nutrients resulting in early ischaemic damage that may favour atherosclerosis. Added to this a reduced arterial distensibility enhances the traumatic effect of blood pressure on the vascular wall, which also leads to an enhanced formation of atherosclerotic lesions as shown in rabbits in which carotid artery expansion is prevented by encasting the vessel into a rigid cuff [14]. Nevertheless, important data are still to be collected in a large diabetic population to give our results a more clear clinical prospective. These include (i) normal values for arterial distensibility and wall thickness, (ii) the rate of progression of diabetic-related abnormalities in arterial distensibility and wall thickness and (iii), a possible delay in progression by controlling either serum glucose profile or by lowering blood pressure or both.

\section{References}

1. Kuller L, Tonascia S (1971) A follow-up study of the commission on chronic illness morbidity survey in Baltimore: IV. Factors influencing mortality from stroke and arteriosclerotic heart disease (1954-1967). J Chronic Dis 24: 111-124

2. Barret-Connor E, Orchard T (1984) Diabetes and heart disease. In: Harrris N (ed) Diabetes in America. NIH, Washington, XVI: 1-41

3. Kannel WB, McGee DL (1979) Diabetes and glucose tolerance as risk factors for cardiovascular disease: The Framingham Study. Diabetes Care 2: 120-126

4. Sawers JR, Ebstein M (1995) Diabetes mellitus and associated hypertension vascular disease and nephropathy, an update. Hypertension 26 (part 1): 869-879

5. Dorman JS, LaPorte RE, Kuller LH et al. (1984) The Pittsburgh insulin-dependent diabetes mellitus (IDDM) morbidity and mortality study. Mortality results. Diabetes 33: 271-276

6. DERI Mortality Study Group (1991) International evaluation of cause-specific mortality and IDDM. Diabetes Care 14: 55-60

7. Kròlewski AS, Kosinsky EJ, Warram JH et al. (1987) Magnitude and determinants of coronary artery disease in juvenile-onset insulin-dependent diabetes mellitus. Am J Cardiol 59: 750-755

8. Morrish NJ, Stevens LK, Head J, Fuller JH, Jarret RJ, Keen H (1990) A prospective study of mortality among middle-aged diabetes patients (the London cohort of the WHO Multinational Study of Vascular Disease in Diabetics) 11: Associated risk factors. Diabetologia 33: 542-548
9. Manson JE, Colditz GA, Stampfer MJ et al. (1991) A prospective study of maturity-onset diabetes mellitus and risk of coronary heart disease and stroke in woman. Arch Intern Med 151: 1141-1147

10. Ferranini E., Hoffner SN, Mitchell BD, Stern MP (1991) Hyperinsulinaemia: the key feature of a cardiovascular and metabolic syndrome. Diabetologia 34: 416-422

11. Jensen T, Borch-Johnsen K, Kofoed-Enevodsen A, Deckert T (1987) Coronary heart disease in young type I (insulin-dependent) diabetic patients with and without diabetic neuropathy: incidence and risk factors. Diabetologia 30: 144-148

12. Beach KW, Brunzell JD, Conquest LL Strandress DF (1979) The correlation of arteriosclerosis obliterans with lipoproteins in insulin-dependent and non-insulin dependent diabetes. Diabetes 28: 836-840

13. Orchard TJ, Dorman JS, Maser RE et al. (1990) Prevalence of complications in IDDM by sex and duration; Pittsburgh Epidemiology of Diabetes Complications Study II. Diabetes 39: 1116-1124

14. Booth RFG, Martin JF, Honey AC, Hassal DG, Beesley JE, Moncada S (1989) Rapid development of atherosclerotic lesions in the rabbit carotid artery induced by perivascular manipulation. Atherosclerosis 76: 257-268

15. Giannattasio C, Mangoni AA, Failla M et al. (1996) Impaired radial artery compliance in normotensive subjects with familial hypercholesterolemia. Atherosclerosis 124: 249-260

16. Wada T, Kodaira K, Fujishiro K et al. (1994) Correlation of ultrasound-measured common carotid artery stiffness with pathological findings. Arterioscler Thromb 14: 479-482

17. Scarpello JHB, Martin TRP, Ward JD (1980) Ultrasound measurements of pulse wave velocity in the peripheral arteries of diabetics subjects. Clin Sci 58: 533-539

18. Wahlqvist ML, Relf IRN, Myers KA, Lo CS (1984) Diabetes and macrovascular disease: risk factors for atherogenesis and non-invasive investigation of arterial disease. Hum Clin Nutr-Clin Phys Physiol Measures 38c: 175-184

19. Lo CS, Relf IRN, Myers KA, Wahlqvist ML (1986) Doppler ultrasound recognition of preclinical changes in the arterial wall in diabetic subjects: compliance and pulse-wave damping. Diabetes Care 9: 27-31

20. Wahlqvist ML, Lo CS, Myers KA, Simpson RW, Simpson IM (1998) Putative determination of arterial wall compliance in NIDDM. Diabetes Care 11: 787-790

21. Hopkins KD, Lehman ED, Jones RL, Turay RE, Gosling RG (1996) A family history of NIDDM is associated with decreased aortic distensibility in normal healthy young adult subjects. Diabetes Care 19: 501-503

22. Lehman Ed Hopkins KD, Gosling RG (1996) Increased aortic stiffness in women with NIDDM. Diabetologia 39: 870-871

23. Salomaa V, Riley W, Kark J, Nardo C, Folson AR (1995) Non-insulin dependent diabetes mellitus and fasting glucose and insulin concentrations are associated with arterial stiffness indexes. The ARIC Study. Circulation 91: 1432-1433

24. Lehmann ED, Gosling RG, Sonksen DH (1992) Arterial wall compliance in diabetes. Diabet Med 9: 114-119

25. Lambert J, Pijpers R, Van Ittersem FJ et al. (1997) Sodium, blood pressure and arterial distensibility in insulin-dependent diabetes mellitus. Hypertension 30: 1162-1168

26. Kool MJF, Lambert J, Stehauwer CDA et al. (1995) Vessel wall properties of large arteries in uncomplicated insulindependent diabetes mellitus (IDDM). Diabetes Care 18: 618-624 
27. Ryden Ahlgren A, Lanne T, Wollmer P, Soressen B, Hansen F, Sundkvist G (1995) Increased arterial stiffness in women, but not in men with IDDM. Diabetologia 38: 1082-1089

28. Christianssen T, Neubauer B (1987) Arterial wall stiffness in insulin-dependent diabetes mellitus. Acta Radiol 28: 207-209

29. Tardy Y, Meister JJ, Perret F, Brunner HR, Arditi M (1991) Noninvasive estimate of the mechanical properties of peripheral arteries from ultrasonic and photoplethysmographic measurements. Clin Phys Physiol Meas 3: 360-367

30. Girerd X, Mourad JJ, Copie X et al. (1994) Noninvasive measurement of medium-sized artery wall thickness in humans: in vitro validation. $\mathrm{J}$ Vasc Res 31 : 114-120

31. Parati G, Casadei R, Groppelli A, Di Rienzo M, Mancia G (1989) Comparison of finger intra-arterial blood pressure monitoring at rest and during laboratory testing. Hypertension 13: 647-655

32. Langewouters GJ, Wesseling KH, Godehart WJA (1984) The static elastic properties of 45 human thoracic and 20 abdominal aortas in vitro and the parameters of a new model. J Biomech 17: 425-435

33. Dobrin PB (1983) Vascular mechanisms. In: Hamilton WF, Dow P (eds) Handbook of Physiology: The Cardiovascular System III, American Physiological Society, Washington, pp 65-102

34. Hoeks A, Brands PJ, Smeets GAM, Reneman RS (1990) Assessment of the distensibility of superficial arteries. Ultrasound Med Biol 16: 121-128

35. Kool MJF, Van Merode T, Reneman RS, Hoeks APG, Struyker Boudier H, Van Bortel LMAB (1994) Evaluation of reproducibility of a vessel wall movement detector system for assessment of large artery properties. Cardiovasc Res 28: 610-614

36. Arcaro G, Laurent S, Jondeau G, Hoeks A, Safar ME (1991) Stiffness of the common carotid artery in treated hypertensive patients. J Hypertens 9: 947-954

37. Salonen R, Haapdnen A, Salonen JT (1991) Measurement of intima-media thickness of common carotid arteries with high-resolution B-mode ultrasonography: inter and intraobserver variability. Ultrasound Med Biol 17: 225-230

38. Mogensen CE, Christensen CK (1984) Predicting diabetic nephropathy in insulin-dependent patients. N Engl J Med 311: 89-93

39. Timini FK, Ting HH, Haley EA, Roddy MA, Ganz P, Creager MA (1998) Vitamin C improves endothelium-depen- dent vasodilation in patients with insulin dependent diabetes mellitus. J Am Coll Cardiol 1,31(3): 552-557

40. Okazaki J, Comori K, Kawasaki K, Eguci D, Ishida M, Sigimaki K (1997) L-arginine inhibits smooth muscle cell proliferation of vein graft intimal thickness in hypercholesterolemic rabbits. Cardiovasc Res 36: 429-436

41. Rudio RD, Shesdy EG, Maeda N, Snithies O, Segal SS, Sessa WC (1998) Direct evidence for the importance of endothelium derived nitric oxide in vascular remodeling. $\mathrm{J}$ Clin Invest 15: 731-736

42. Kekakis JP, Papamichael CH, Vemmos CN, Voutas AA, Stamatelopoulos SF, Molopoulos SD (1998) Peripheral vascular endothelial dysfunction in patients with angina pectoris and normal coronary artheriograms. J Am Coll Cardiol 1: 541-546

43. Ruiz-Torres A, Melon J, Munoz FJ (1998) Insulin stimulates collagen synthesis in vascular smooth muscle cells from elderly patients. Gerontology 44: 144-148

44. Parker SB, Wade SS, Prewitt RL (1998) Pressure mediates angiotensin II-induced arterial hypertrophy and PDGF-A expression. Hypertension 32: 452-458

45. Chakravarthy U, Hayes RG, Stitt AW, Mcawley E, Arker DD (1998) Constitutive nitric oxide synthase expression in retinal vascular endothelial cell is suppressed by high glucose and advanced glycation end products. Diabetes 44: 945-952

46. Litwak KN, Cefalù WT, Wagner JD (1998) Chronic hyperglycemia increases arterial low density lipoprotein metabolism and atherosclerosis in cynomolgus monkeys. Metabolism 47: 947-954

47. Trazzi S, Omboni S, Santucciu C, Parati G, Mancia G (1992) Variability in arterial diameter and compliance: compliance modulation reserve. J Hypertens 10:S41-S43

48. Takizawa S, Ozaki H, Karaki H (1997) Inteurleukin 1 beta induced, nitric oxide-dependent and independent inhibition of vascular smooth muscle contraction. Eur J Pharmacol 330: 143-150

49. Giannattasio C, Rivolta MR, Failla M, Mangoni A, Stella ML, Mancia G (1997) Large and medium sized artery abnormalities in untreated and treated hypothyroidism. Eur Heart J 18: 1492-1498

50. Stella ML, Failla M, Mangoni AA, Carugo S, Giannattasio S, Mancia G (1998) Effects of isolated systolic hypertension and essential hypertension on large and middle-sized artery compliance. Blood Press 7: 96-102 